\title{
CONSIDERACIONES SOBRE LA PUBLICACIÓN DE DICTÁMENES DE LOS COMITÉS DE BIOÉTICA
}

\author{
Irma Alejandra Coronado Zarco ${ }^{1}$, Octaviano Domínguez Márquez²
}

Resumen: Este es un análisis sobre la forma en que la comunicación escrita puede llegar a ser una herramienta de influencia y legitimidad para los comités de ética. Se plantea a partir de una revisión histórica de documentos generados por diversos tipos de comités de ética, que han influido en toma de decisiones trascendentales en el ámbito médico, legal y social, y propone algunos factores a considerar en la estructuración de todo documento generado por los comités a partir de sus actividades, así como el contexto médico, científico, social y legal. Esta revisión y análisis histórico genera una lista de funciones que podrían tener las diversas publicaciones de los comités de bioética.

Palabras clave: comités de ética, publicaciones éticas

\section{Considerations on the publication of decisions by ethics committees}

\begin{abstract}
This is an analysis about the form in which written communication can become a tool of influence and legitimacy for ethics committees. It stems from a historical revision of documents generated by diverse types of ethics committees which have had an influence on transcendental decisions on the medical, legal, and social spheres and proposes some factors to be considered in every document generated by committees in their activities as well as the scientific, medical, social, and legal contexts. This review and the historical analysis generate a list of functions for the diverse publications of ethics committees
\end{abstract}

Key words: ethics committees, ethics publications

\section{Consideraçóes sobre a publicaçáo de pareceres dos comitês de bioética}

Resumo: Esta é uma análise sobre a forma pela qual a comunicação escrita pode chegar a ser uma ferramenta de influência e legitimidade para os comitês de ética. É suscitada a partir de uma revisão histórica de documentos gerados por diversos tipos de comitês de ética, que influiram na tomada de decisóes transcendentais no âmbito médico, legal e social, e se propóe alguns fatores a serem considerados na estruturação de todo documento gerado pelos comitês a partir de suas atividades, no contexto médico, científico, social e legal. Esta revisão e análise histórica geram uma lista de funçóes que poderiam ter as diversas publicaçóes dos comitês de bioética.

Palavras-chave: comitês de ética, publicações éticas

\footnotetext{
${ }^{1}$ Médica Pediatra Neonatóloga. Unidad de Cuidados Intermedios al Recién Nacido del Instituto Nacional de Perinatología Isidro Espinosa de los Reyes SSA, México

Correspondencia: ucincmn20@hotmail.com

${ }^{2}$ Médico Cirujano Pediatra. Escuela Superior de Medicina, Instituto Politécnico Nacional, México
} 
De acuerdo con Gabriel Zaid, el protagonismo que puede alcanzar un individuo en su comunidad dependerá de manera fundamental de su papel social, su carácter personal y también del número de participantes en la comunidad donde se desempeña(1). Podríamos decir que todo ello depende de su capacidad para comunicarse. Comunicar es hacer partícipe de algo a otros; es vincular al ser humano con el mundo que lo rodea y con los demás seres humanos.

Antaño, los comités de ética institucionales se creaban básicamente para revisar decisiones sobre limitación o retiro de tratamiento de sostén para la vida, en caso de pacientes dañados neurológicamente o en fase terminal. Sin embargo, su rol ha evolucionado con el paso del tiempo. Hoy constituyen parte del proceso de implementación de regulaciones federales, resolución de conflictos sobre decisiones terapéuticas, foros para discusión de políticas relacionadas con la ética institucional y educación a la comunidad (no solo la relacionada con la salud, sino la población en general) sobre asuntos éticos(2). Es así que los comités pueden considerarse sitios de oportunidad para la vinculación de la bioética con su comunidad y con el entorno sobre el que realiza su reflexión. No son esencialmente estructuras con poder, pero pueden lograr su legitimidad institucional y social con una actividad competente, evidenciando compromiso, responsabilidad, efectividad y eficacia. Dadas sus funciones consultivas, normativas y educativas, el documento escrito es una de las formas más efectivas de difundirlas.

A lo largo de la historia, los hombres han contado con estructuras morales de diversa índole. Cada una creada de acuerdo con las necesidades del propio grupo humano en el que es generada. Dichas estructuras morales han dejado evidencia escrita de sus preceptos, de sus códigos de conducta e incluso reflexiones sobre las mismas, aun cuando la mayoría de los integrantes de sus comunidades no sabía leer. Ejemplo pueden ser el códice de Ur-Nammu del Rey Ur, 2050 años antes de Cristo; el códice de Eshnunna 1930 a.C.; el códice Lipit-Ishtar 1870 a. C. y el código de Hammurabi 1780 a.C. En las culturas orientales, el Charaka Samhita, uno de los principales textos sobre salud y vida del Ayuveda, también enfatiza algunos valores en torno a los seres humanos(3). Probablemente, el código más famoso es el hipocrático, en el siglo IV a.C(4). Moisés Maimónides ejerció gran influencia filosófica en Tomás de Aquino, Alberto Magno y Averroes a través de sus escritos. Thomas Percival publica "Ética Médica" en 1794, que a la fecha es la base para el código de la Asociación Americana de Medicina(5). La misma historia nos da muestra fehaciente de que para trascender es necesario dejar constancia. La forma más efectiva es la escrita.

Uno de los mejores ejemplos de las implicaciones de la publicación de los dictámenes de un comité de bioética es el documento generado en el Comité $A d$ Hoc de la Escuela de Medicina de Harvard para examinar la definición de "muerte cerebral" (Ad Hoc Committee of the Harvard Medical School to Examine the Definition of Brain Death)(6). Este texto es considerado mundialmente un punto de referencia fundamental para el establecimiento del concepto de "muerte cerebral". Diversos historiadores y bioeticistas se han referido a este reporte como un intento de expandir la práctica de trasplantes y eliminar las menciones inadecuadas de los cuestionamientos morales auténticos, con el fin de sustituirlos por aquellos generados por expertos en medicina. Tina Stevens publicó: "más que una respuesta médica al problema moral generado tecnológicamente, "muerte cerebral" es un artificio para autoprotección legal...". Robert Veatch, eventual director del Centro Kennedy para la Ética (Kennedy Center for Ethics), criticó al comité de la siguiente manera: "la labor de definir la muerte no es un ejercicio trivial para acuñar el significado del término. En su lugar, es un intento de alcanzar la comprensión de la naturaleza filosófica del hombre. Dejar dicha decisión en manos de profesionales entrenados científicamente es un movimiento peligroso" (7).

Sin embargo, la mayoría de este tipo de críticas ha surgido sin una revisión y análisis detallado del reporte generado por el comité. Repasar el documento muestra la forma sistematizada y cuidadosa en que Henry Beecher, director del comité y jefe de anestesiología del Hospital General de Massachusetts, con el neurólogo Robert Schwab, 
organizaron dicho comité. En el documento se muestra que existía ya con anterioridad un importante trabajo de investigación y reflexión ética que sirvió de sustento para la emisión de los criterios. Se alude a que una de las principales preocupaciones del comité era la incertidumbre en que se encontraban aquellas personas en estado de coma, mantenidas con vida artificialmente, como quedó evidenciado en la reunión del Comité de Estudios Humanos de la Escuela de Medicina de Harvard (Standing Committee of Human Studies), a la que convocó Beecher previo a la integración del comité a su cargo. El título de su ponencia fue en aquella ocasión: "Problemas éticos creados por el paciente inconsciente sin esperanza" (7). Si bien el reporte puede ser objeto de diversas críticas, las opiniones deberían ser sustentadas en la lectura detenida del escrito.

Otro ejemplo de los alcances de documentos éticos publicados es el caso de Karen Quinlan, quien quedó en coma profundo conectada a un respirador artificial por 13 meses. Los padres solicitaron a la Suprema Corte de Justicia de Nueva Jersey, en Estados Unidos de América, que les fuera permitido desconectarla, ya que las autoridades del hospital donde se encontraba se negaban a hacerlo. Finalmente, el juez Richard Hughes autorizó la desconexión en 1976, falleciendo Karen hasta nueve ańos después (8). Durante el sustento de su fallo, el juez citó como una de las más importantes influencias en esta decisión un artículo publicado por la pediatra norteamericana Karen Teel, en 1975, quien propuso la creación de comités de ética para contribuir a la resolución de este tipo de dilemas(9). Es así que fue creado un comité hospitalario para el caso de Karen, que si bien ha sido criticado por la forma en que se estructuró y funcionó, jugó un rol fundamental en la decisión final de la corte. Hoy la evidencia escrita en torno a dicho caso nos permite analizarlo y aprender de él.

Revisando la práctica de comités de bioética en el plano internacional, podemos encontrar al Comité Internacional de Bioética, creado dentro de la UNESCO (Organización Educacional, Científica y Cultural de las Naciones Unidas) en 1993. Dicho comité cuenta con una página en internet desde la cual se puede acceder a los reportes de sus sesiones, los estatutos, las conclusiones, diversas publicaciones, sus metas, objetivos, programas de acción, su composición y organización del trabajo(10).

Espace Éthique es una estructura autodefinida como de asistencia pública en el Hospital de París (Hóspitaux de Paris)(11). Es un lugar de intercambio, enseñanza, formación, investigación, evaluación y proposición en el ámbito de la ética hospitalaria, y una fuente de generación de recursos documentales. Se encuentra vinculado al Comité Consultivo Nacional de Ética (CCNE Comité Consultatif National D'Etique) en Francia, que anualmente emite un reporte sobre actividades, decretos y/o dictámenes(12).

La mayoría de los comités de bioética organizados tienen como una de sus actividades establecidas la publicación de sus dictámenes, posturas sobre diversos dilemas éticos y su forma de organización. ¿Qué debe contener un reporte de comité de ética? La respuesta varía de acuerdo a sus objetivos, su organización y los propósitos de la publicación.

Un análisis de los reportes anuales de los comités de ética de investigación local en Inglaterra da cuenta de que incluyen el nombre de los miembros del comité, el número de reuniones que realizaron y una lista de los puntos analizados, incluido el resultado de dichos análisis (aprobado, desaprobado, aprobado con modificación, rechazado o eliminado). Basados en la calidad de las publicaciones, los autores consideraron factible calificar el trabajo de los comités, ya que asumieron que dichos documentos son un reflejo claro de la seriedad y compromiso de sus miembros (13).

La Sociedad para la Salud y Valores Humanos. Equipo de Trabajo para Consultoría de la Sociedad de Bioética en Estados Unidos, plantea que la documentación de las consultorías realizadas a los comités debe quedar plasmada en el expediente del paciente o en algún otro registro permanente. Los resultados que éticamente requieren involucrar a los pacientes deben ser comunicados a éstos. Idealmente, debe existir una política espe- 
cificando el grado y tipo de documentación que requiere cada consulta. Dicha documentación optimiza la comunicación, facilita la mejoría en la calidad de atención y formaliza la responsabilidad de los comités (14).

Entre los dilemas que surgen de la publicación de los reportes de los comités de bioética está el derecho a la privacidad. Hoy la mayoría de los países se encuentran legislando sobre la libertad y acceso a la información. Ello significa que, salvo que la legislación lo estipule, estos reportes podrán ser accesibles para cualquier persona, con las implicaciones que ello conlleva. La elaboración de dichos documentos tendrá que ser definida cuidadosamente para hacer compatible los derechos de los pacientes y las disposiciones legales(15). Equilibrar la libertad de acceso informativo con la privacidad fundamental de los individuos es hoy uno de los grandes retos, especialmente cuando los principales despliegues tecnológicos se ha enfocado en la comunicación. Si bien el aporte tecnológico al desarrollo de la comunicación es trascendental, se debe considerar que el proceso per $s e$ ha generado vulnerabilidad en diversos puntos relacionados con la intimidad de los individuos. Ello debe ser considerado por los comités éticos al momento de definir lo público y lo privado, así como los recursos de difusión que utilizarán. Para un manejo adecuado de la información y la definición de procedimientos en torno a la misma, es fundamental que se favorezca la capacitación de los miembros del comité en este ámbito. De acuerdo con las guías de Comités de Bioética de la UNESCO, debe quedar asentado un programa de capacitación permanente para los miembros del comité que incluya este punto ${ }^{10}$.
Los documentos publicados por los Comités de Bioética pueden tener las siguientes funciones:

- Ubicarse como punto de referencia para normatividad hospitalaria.

- Situarse como punto de referencia en el marco jurídico.

- Consolidarse como fuente de información para acciones legales.

- Constituirse como elementos para evaluación de comités.

- Constituirse como elementos para evaluación de instituciones.

- Facilitar la transparencia en el ejercicio de los comités de bioética.

- Ser un importante recurso para obtener autoridad moral dentro de la comunidad.

- Coadyuvar en la creación de antecedentes históricos.

- Contribuir a hacer más eficiente la comunicación entre el comité de bioética y la comunidad donde se encuentra inserto.

- Facilitar la comunicación entre diversos comités para enriquecer sus capacidades.

- Hacer partícipe de los asuntos bioéticos a la comunidad.

- Constituirse como un recurso educativo para el propio comité y otros.

En nuestra opinión, la publicación del trabajo de los comités de bioética, con cierta regularidad y sistematización, contribuirá a su mejor desempeńo, ya que facilitará la retroalimentación y la interacción con otros comités y con la comunidad en la que se encuentra inserto. Sin embargo, se debe ser cauteloso en las formas de manejo de la información, para ser consecuente con uno de los objetivos fundamentales de dichos comités: proteger la integridad y derechos de los individuos. 


\section{Referencias}

1. Zaid G. Economía del protagonismo. Letras Libres 2004; 61: 64-65.

2. American Academy Pediatrics. Institutional Ethics Committees. Pediatrics 2001; 107(1): 205-209.

3. Baker JR. Women's Rights in Old Testament Times. Signature Books, Salt Lake City, 1992. Disponible en http://signaturebookslibrary.org/?p=5380 (Acceso en 2011 Jun 28)

4. Zerón JA, Ladrón de Guevara F. Código de Ética de la ADM. Aceptado en Asamblea General ADDF y ADM para ser adoptado por la Asociación Dental Mexicana AC, 1996 Disponible en http://www.adm.org. mx/ckfinder/userfiles/files/codigo-etica.pdf (Acceso en 2011 Mar 29)

5. American Medical Association. Principles of medical ethics, 2001 Disponible en http://www.ama-assn.org/ ama/pub/physician-resources/medical-ethics/code-medical-ethics/principles-medical-ethics.page? (Acceso en 2011 feb 5)

6. Brain Death. A Definition of Irreversible Coma-Report of the Ad Hoc Committee of the Harvard Medical School to Examine the Definition of Brain Death. J Am Med Assoc 1968; 205: 337-340.

7. Belkin GS. Brain Death and the Historical Understanding of Bioethics. Journal of the History of Medicine and Allied Sciences 2003; 58: 325-361.

8. Pérez A. Karen Quinlan respira sin ayuda. El País, 26/05/1976. Disponible en http://www.elpais.com/articulo/sociedad/Karen/Quinlan/respira/ayuda/elpepisoc/19760526elpepisoc 29/Tes/ (Acceso en 2011 Jan 20)

9. Teel K. The physician's dilemma: a doctor's view-what the law should be. Bayl Law Rev 1975; 27; 6-9

10. International Bioethics Committee (IBC). United Nations Educational, Scientific and Cultural Organization, 2011. Disponible en http://www.unesco.org/new/en/social-and-human-sciences/themes/bioethics/ international-bioethics-committee/ (Acceso en 2011 Jul 1)

11. Espace éthique. Hóspitaux de Paris, 2011 Disponible en http://initiative-ethique.fr/espace-ethiqueap-hp/ (Acceso en 2011 Jan 10)

12. Comité Consultatif National d'Éthique. Pour les sciences de la vie et de la santé, 2009 Disponible en http:// www.ccne-ethique.fr (Acceso en $2011 \mathrm{Feb} 3$ )

13. Foster CG, Marshall T, Moodie P. The annual reports of local research ethics committees. Journal of Medical Ethics 1995; 21: 214-219.

14. Aulislo MP, Arnold RM, Youngner SJ. Society for Health and human Values-Society for Bioethics Consultation Task Force on Standards for Bioethics Consultation. Health Care Ethics Consultation: Nature, goals and competencies. Ann Intern Med 2000; 133: 59-69.

15. Eike-Henner K. The roles and funtions of hospital-based ethics committees. Can Med Assoc J 1996; 154: 91094-91095.

Recibido: 22 de agosto de 2011

Aceptado: 18 de noviembre de 2011 